\title{
The Kitt Peak Electron Multiplying CCD demonstrator
}

\author{
Michael W. Coughlin ${ }^{\odot},{ }^{1 \star}$ Richard G. Dekany, ${ }^{2}$ Dmitry A. Duev, ${ }^{1}$ Michael Feeney, ${ }^{2}$ \\ S. R. Kulkarni, ${ }^{1,2}$ Reed Riddle ${ }^{\odot},{ }^{2}$ Tomás Ahumada, ${ }^{3}$ Kevin Burdge,${ }^{1}$ Alison M. Dugas, ${ }^{1}$ \\ Christoffer U. Fremling, ${ }^{1}$ Gregg Hallinan, ${ }^{1}$ Thomas A. Prince ${ }^{\odot 1}$ and Jan van Roestel ${ }^{\oplus 1}$ \\ ${ }^{1}$ Division of Physics, Math, and Astronomy, California Institute of Technology, Pasadena, CA 91125, USA \\ ${ }^{2}$ Caltech Optical Observatories, California Institute of Technology, Pasadena, CA 91125, USA \\ ${ }^{3}$ Department of Astronomy, University of Maryland, College Park, MD 20742, USA
}

Accepted 2019 February 13. Received 2019 February 13; in original form 2019 January 14

\begin{abstract}
The Kitt Peak Electron Multiplying CCD (EMCCD) demonstrator is a new instrument that has been developed for use at the Kitt Peak National Observatory's 84-inch telescope. The EMCCD enables single-band optical imaging in the Sloan $g$ and $r$ bands and Johnson UVRI filters. The EMCCD is chosen for its sub-electron effective read noise using large multiplicative gains. With these systems, frame rates of greater than $1 \mathrm{~Hz}$ are possible. The field of view is $4.4 \times 4.4 \operatorname{arcmin}^{2}$ and the pixel size is 0.259 arcsec. This camera, coupled with a fully roboticized telescope, is ideal for follow-up of short period, white dwarf binary candidates, as well as short duration transient and periodic sources identified by large field-of-view all-sky surveys such as the Zwicky Transient Facility.
\end{abstract}

Key words: instrumentation: photometers - techniques: photometric.

\section{INTRODUCTION}

One of the motivations for the current generation of all-sky surveys are the study of variable and transient sources. Examples of current and future wide-field telescopes include the Panoramic Survey Telescope and Rapid Response System (Pan-STARRS; Morgan et al. 2012), the Asteroid Terrestrial-impact Last Alert System (ATLAS; Tonry et al. 2018), the All-Sky Automated Survey for SuperNovae (ASAS-SN; Shappee et al. 2014; Kochanek et al. 2017), the Zwicky Transient Facility (ZTF; ; Graham et al. 2018; Bellm et al. 2019), and the Large Synoptic Survey Telescope (LSST; Ivezic et al. 2008). The study of variability, in particular, is an important part of studying stellar and galactic structure and evolution. The current generation of astronomical surveys, with a variety of cadences, sensitivities, and fields of view (FOVs), is resulting in a variety of time-scales to study variable objects. ZTF, in particular, with its $\sim 47 \mathrm{deg}^{2}$ field of view and a range of cadences ( $\sim 3 \mathrm{~d}-$ minutes) is finding variables on time-scales from months to minutes.

The Kitt Peak Electron Multiplying CCD (EMCCD) demonstrator (KPED), consisting of an EMCCD behind a filter wheel imager located at the Cassegrain focus of the Kitt Peak 84-inch (KP84) telescope, is designed for rapid and sensitive photometric followup of variable and transient sources identified by these surveys. CHIMERA (Harding et al. 2016), mounted on the Palomar 200inch and observing simultaneously in two filters, and ULTRACAM

\footnotetext{
^E-mail: mcoughli@caltech.edu
}

(Dhillon et al. 2007), mounted on the William Herschel Telescope and observing simultaneously in three filters, serve similar purposes.

KPED combines two important technologies: EMCCDs and robotic telescopes. With robotic telescopes, one can optimize observations to be near zenith, minimizing the observation time required. EMCCDs use a 'high gain' or electron multiplication(EM) register, which is a second stage of readout that amplifies electrons in a process known as 'avalanche multiplication'. The low noise characteristics and rapid readout speeds, allowed by the extremely low noise $\left(<1 \mathrm{e}^{-} \mathrm{rms}\right)$ and high-speed clocking of the EMCCDs, makes this mode possible. At the cost of pixel-charge capacity, much higher signal-to-noise ratios (SNRs) are possible above and beyond conventional CCD or CMOS detectors. The large FOV of the instrument is derived from a sensor of $1024 \times 1024$ pixels with a plate scale of $0.26 \mathrm{arcsec} \mathrm{pixel}^{-1}$, resulting in a $4.4 \times 4.4 \mathrm{arcmin}^{2}$ FOV. The instrument has sufficient FOV to allow for differential photometry from field stars.

Most EMCCDs use a frame-transfer design, where half of the chip is covered with an opaque layer such that it can serve as a storage unit for the device. The charge is transferred rapidly into the storage area, where it is readout as the next image is taken. This frame transfer means that observing efficiency is greatly improved. There are some downsides to this technology. For example, the higher frame rates means that subtle noise sources such as charge transfer inefficiency are more visible than in conventional CCDs. There are also EMCCD specific noise sources, such as multiplication noise (Tulloch \& Dhillon 2011). By design, a photoelectron entering the EM register can potentially result in a wide range of output signals. For this reason, for a given signal measured, many possible input signals are 
generally possible. This has the effect of doubling the variance of the signal, which is statistically equivalent to reducing the quantum efficiency of the camera by a factor of two. Clock-induced charge, also present in conventional CCDs but often ignored, comes into play for these systems as well.

We now highlight a few science cases for this system. White dwarf (WD) stars (also known as degenerate dwarfs) are the final stages of evolution of stars with initial masses below approximately 7-9 $\mathrm{M}_{\odot}$, found in both single and binary systems (Dobbie et al. 2006). WD binaries, emitting gravitational waves and thereby losing orbital angular momentum, are one of the most common sources for the future Laser Interferometer Space Antenna mission (LISA; Breivik et al. 2018). When these objects merge, they are expected to form a variety of exotic objects, including hydrogen-deficient stars such as R Coronae Borealis stars, single subdwarfs, and Type Ia supernovae (Iben \& Tutukov 1984; Webbink 1984; Saio \& Jeffery 2002). Previous measurements of WD binaries have resulted in determinations of the WD mass-radius relationship (Hermes et al. 2014). Eclipses can constrain orbital eccentricities, mass ratios of the binaries (Kaplan 2010), and the orbital evolution (Fuller \& Lai 2011). Due to their potentially small periods $(\approx 1 \mathrm{~h})$, KPED, with its ability to do rapid and sensitive photometry, is particularly useful for identifying and characterizing WD binaries. Other periodic sources that can be targeted include aurorae on brown dwarfs, transiting planets, flaring stars, and eclipsing binaries.

The detection of GW170817 (Abbott et al. 2017) by the Advanced LIGO (Aasi et al. 2015) and Advanced Virgo (Acernese et al. 2015) GW detectors ushered in a new era of multimessenger astronomy when electromagnetic facilities observed a short gamma-ray burst (Savchenko et al. 2017) and a kilonova (Kilpatrick et al. 2017) in coincidence with the gravitational-wave (GW) detection. To facilitate counterpart detection, many wide-field optical telescopes are expected to image the sky localization regions of GW and Fermi Gamma-ray Burst Monitor (GBM) triggers. Due to the poor localizations, many potential candidates will be detected. For this reason, KPED is well suited to follow-up of transients identified by other telescopes for the purpose of identification and classification of optical counterparts to GW or short gamma-ray burst (SGRB) events.

The goal for this paper is to present the design and performance of the KPED instrument. Section 2 gives the instrument design. We describe performance of the telescope and camera system in Section 3. We present first light science results from the system in Section 4. We present our conclusions and paths for future work in Section 5.

\section{INSTRUMENT AND SOFTWARE DESIGN}

\subsection{Instrument}

KPED is mounted on the backside of the KP84 primary mirror cell with a stand-off structure at the Cassegrain $f / 7.6$ focal plane (Fig. 1). The stand-off structure, adapted from Robo AO (JensenClem et al. 2018), provides a stiff mounting surface in the correct focus position $(\approx 0.4 \mathrm{~m}$ from the primary cell). Sitting adjacent to the instrument is a single electronics rack which houses all of the necessary support equipment (e.g. control computer, network power switch, power/data cables, etc.).

KPED is comprised of primarily off-the-shelf components and a few custom manufactured parts. The instrument structure is shown on the left-hand panel of Fig. 2. A new instrument mounting plate was manufactured for KPED to adapt to the existing instrument

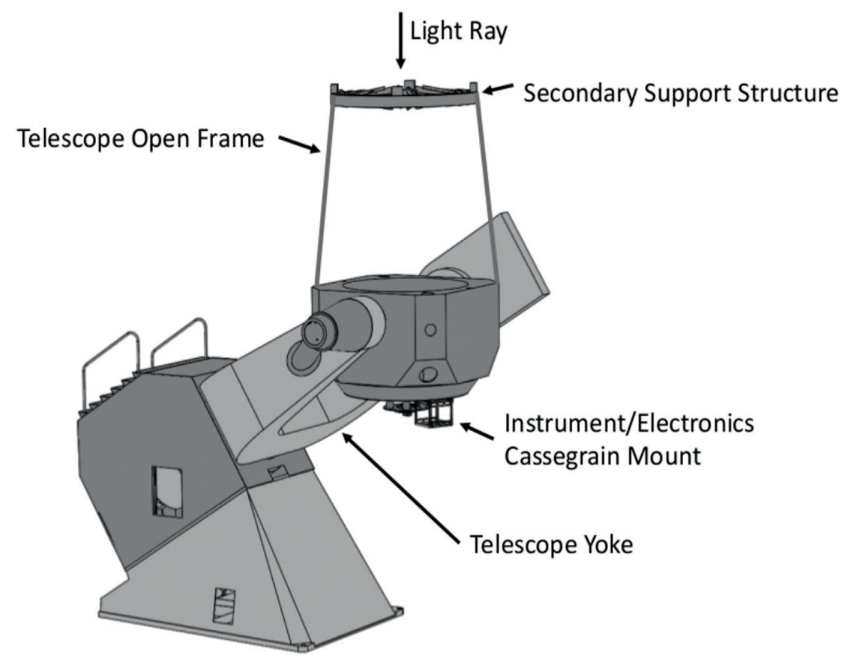

Figure 1. KPED and electronics rack shown in situ with the 84-inch telescope.

stand-off structure. This plate provides a rigid mounting surface for the camera assembly and tip/tilt/piston plate. Adjustment of tip, tilt, and piston is achieved by adjusting the length of three 1/2-in. 13 threads/in. bolts with a jam nut and spherical washer stack-up. The jam nuts lock the position down and the spherical washers provide a flat, compensating interface. Fine lateral adjustment is achieved through three Newport nudgers at the corresponding bolt interfaces. The right-hand panel of Fig. 2 shows the instrument in an assembled state. We show a photograph of the system in Fig. 3.

The camera is an Andor iXon 888 unit; the detector is an array of $1024 \times 1024$ pixels with a pixel size of $13 \mu \mathrm{m}$. The camera is cooled by thermoelectric cooling to reach $-80^{\circ} \mathrm{C}$. We highlight a selection of system specifications and performance parameters in Table 1. With the use of 1/4-in.-20 threads/in. stand-offs and custom L-brackets, the iXon 888 is fixed to the tip/tilt/piston plate. The Lbrackets are slotted for additional focus adjustment. The front of the iXon 888 has a C-Mount (1-in.-32 threads/in) for mounting various fore optics. KPED utilizes the C-Mount to constrain a Finger Lakes Instruments (FLI) Filter Wheel (part number CFW-3-10) and a Newport PAC087 Barlow lens. In the filter wheel, the selectable filters are Sloan $g$, Sloan $r$, and Johnson UVRI. The Sloan filters are the SDSS Gen2 filters from Astrodon. Additionally, there are two empty slots in each of the overlapping wheels for a clear path to the detector and a plastic blank for darks. To mate the filter wheel to the camera, a custom C-Mount to the FLI Zero Tilt Mount adapter was manufactured by Precise Parts. On the front face of the filter wheel is a Newport 2-in.-40 threads/in. lens tube, which houses the Barlow lens. Precise Parts provided a similar adapter to make this connection. The light provided from the telescope first enters the PAC087 lens fore optic, then travels through the selected filter, and finally lands on to the iXon 888 detector. There are two cables to connect the electronics rack to the camera, a USB for communications and control and a Camera Link (Andor) for transferring image data.

\subsection{Software}

KPED's control software, written in $\mathrm{C}++$ and compiled with $g c c++$, consists of three main tasks. The first is the telescope control software, the second is the camera control, and the third is the filter wheel control. All control software is run through terminals, 

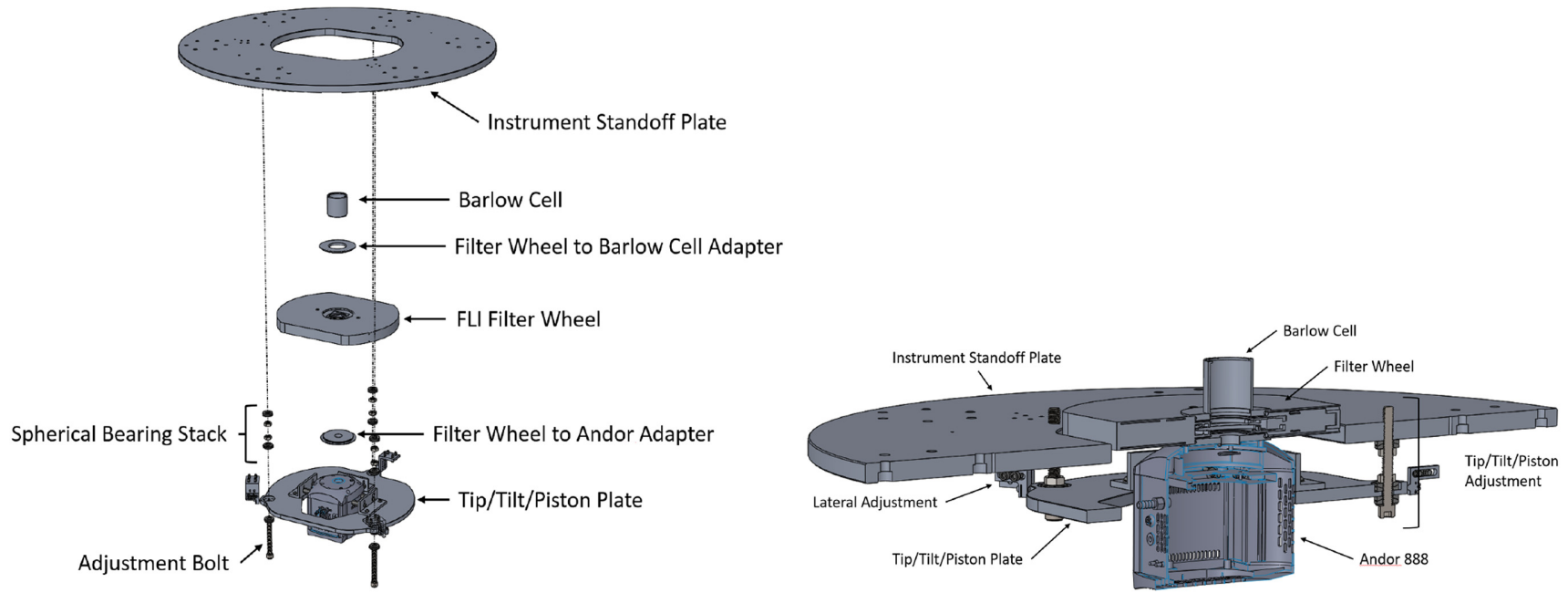

Figure 2. Instrument diagrams for KPED with the instrument breakdown exploded view on the left-hand panel and the assembled view on the right-hand panel.

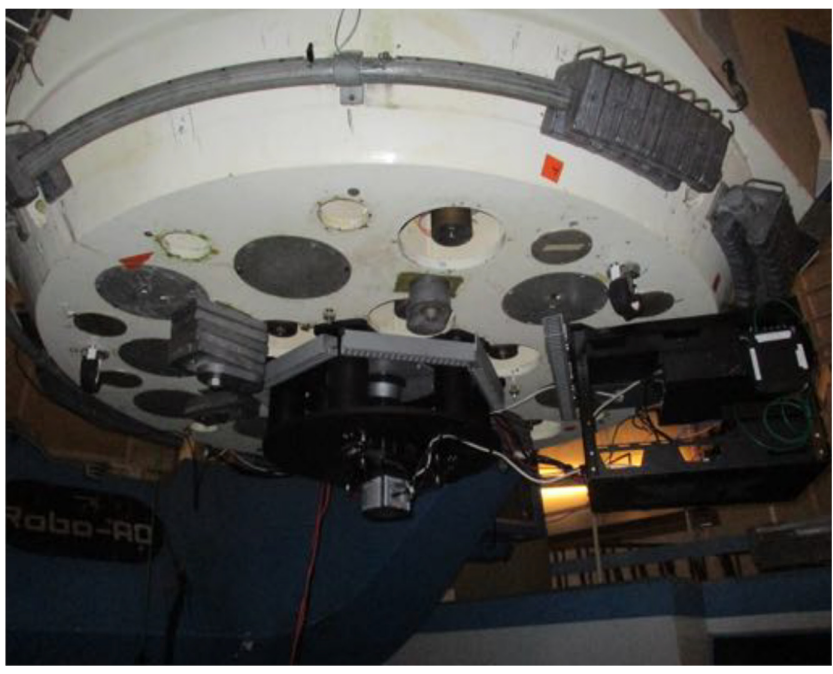

Figure 3. Photograph of KPED and electronics rack installation.

allowing for straightforward remote observations. It is based on ROBO-AO software (Riddle et al. 2012) and includes improvements developed for ZTF (Dekany et al. 2018).

The camera control software relies on Andor's software development kit (2.102.30034). The software interfaces with the camera through the CameraLink frame grabber PCIe card for image acquisition installed in the control computer. The camera is run in two modes, one with the conventional amplifier and the other with the EM amplifier. Within the EMCCD mode, five configurations are currently available, with EM gains ranging from 2-300. The results in the following sections use an EM gain of 300. Currently, only $1 \times 1$ binning is possible, and no sub-framed modes are supported. We plan to implement these modes in the future. The camera buffer is configured such that the oldest image is retrieved first, known as a 'first in, first out' buffer.

The filter wheel control software relies on Finger Lakes Instrument's software development kit (1.104). The software requests positions by a numerical index of each of the two filter wheels. The numerical index is mapped to the filters using a configuration file.
Table 1. Andor iXon Ultra 888 system specifications and performance parameters. The read noise parameters are for the modes routinely used in observations. While the gain range can reach 1000, we do not use it in observations for safety reasons.

\begin{tabular}{lc}
\hline Parameter & Specification \\
\hline Active pixels (image) & $1024 \times 1024$ \\
Pixel size & $13 \mu \mathrm{m}$ \\
Utilized gain range & $1-300$ \\
Digitization (Conv. horiz.) & $16-\mathrm{bit}$ \\
Digitization (EM horiz.) & $14-\mathrm{bit}$ \\
Read-out rate (Conv. horiz.) & $1,0.1 \mathrm{MHz}$ \\
Read-out rate (EM horiz.) & $10,5,3,1 \mathrm{MHz}$ \\
Dark current $\left(-100^{\circ} \mathrm{C}\right)$ & $6.6 \times 10^{-5} \mathrm{e}^{-} \mathrm{pix}^{-1} \mathrm{~s}^{-1}$ \\
Read noise (Conv., 1 MHz) & $\sim 6 \mathrm{e}^{-} \mathrm{rms}$ \\
Read noise (EM, 10 MHz) & $\sim 46 \mathrm{e}^{-} \mathrm{rms}$ \\
Eff. read noise (w/EM gain) & $<1 \mathrm{e}^{-} \mathrm{rms}$ \\
\hline
\end{tabular}

The telescope control system software handles the remaining requirements for the system. The software governs both the dome and the telescope and is fully integrated into the telescope control software. Control of telescope is currently given by selection of RA and Declination for sources. No guiding is currently available. Due to the high frame rates of the science images, in principle, they can be used for guiding. Guiding software will be developed based on the new ZTF guiding system.

KPED's data reduction software is based upon the automated data reduction and archiving system of ROBO-AO (Jensen-Clem et al. 2018). The automated 'house-keeping' (HK) system uses a distributed task processing queue based on the dask. distributed python module. ${ }^{1}$ The processing results, together with ancillary information on individual observations, are stored in a MongoDB NoSQL data base. ${ }^{2}$ The whole HK system is containerized using DOCKER software. ${ }^{3}$

The data reduction chain for an observing night proceeds as follows. At the end of each night, the EMCCD data are compressed

\footnotetext{
${ }^{1}$ https://github.com/dask/distributed

${ }^{2}$ https://www.mongodb.com/

${ }^{3}$ https://www.docker.com/
} 
Table 2. KPED astrometric solution.

\begin{tabular}{lccc}
\hline FITS WCS keyword & Value & Standard Deviation & Unit \\
\hline CD1_1 & $-7.120 \times 10^{-5}$ & $6 \times 10^{-8}$ & deg pix $^{-1}$ \\
CD1_2 & $5.0 \times 10^{-7}$ & $8 \times 10^{-8}$ & deg pix $^{-1}$ \\
CD2_1 & $5.3 \times 10^{-7}$ & $6 \times 10^{-8}$ & deg pix $^{-1}$ \\
CD2_2 & $7.121 \times 10^{-5}$ & $8 \times 10^{-8}$ & deg pix $^{-1}$ \\
CRPIX1 & 512.019 & 0.003 & pix \\
CRPIX2 & 512.002 & 0.002 & pix \\
CCD_ROT & 0.423 & 0.045 & deg \\
PIXEL_SCALE1 & 0.2563 & 0.0002 & $\operatorname{arcsec~pix~}^{-1}$ \\
PIXEL_SCALE2 & 0.2563 & 0.0003 & $\operatorname{arcsec~pix~}^{-1}$ \\
\hline
\end{tabular}

and transferred to the network storage. The darks and dome flats taken at the beginning of each night are then combined into master calibration files and applied to the observations.

The next step is the image registration pipeline. The first five frames from an observation are median combined to avoid selecting cosmic rays as guide stars. The resulting 'master' reference image is high-pass filtered and windowed about an automatically selected guide star. Each raw short exposure frame is then dark and flat corrected, high-pass filtered, and windowed. We use the IMAGE REGISTRATION FOR ASTRONOMY 4 package to register the individual frames to the master reference frame, thereby accounting for telescope drift and pointing errors. This is accomplished by using a cross-correlation based subpixel image translation registration, followed by an FFT-based subpixel image shift. The pipeline outputs both the FITS-cubes with individually aligned frames as well as a stacked image.

Next, the images are registered to sky coordinates. The astrometric pipeline proceeds as follows. We detect individual sources in the stacked image and extract their pixel positions using the SEXTRACTOR software package. We proceed if a sufficient number of sources has been detected. At the moment, we require at least 12 sources. This is more than the five independent parameters solved for, corresponding to the RA and Declination of the FOV centre, the scale factors in both axes, and theotation (see Table 2). This is currently a conservative choice, and we will explore relaxing this requirement going forward. To identify the sky positions of the extracted sources, we take advantage of the precise astrometry provided by the Gaia Mission Data Release 2 (DR2) catalogue (Gaia Collaboration 2016, 2018). We query the DR2 catalogue within 200 arcsec of the centre of an image (recorded by the 84inch telescope control system in the 'TELRA' and 'TELDEC' FITS header keywords). Next, we cross-match the sources extracted from our image with the Gaia sources. This is accomplished by first generating a coarse a priori astrometric solution to project the Gaia star positions on to the detector plane. We then generate a synthetic image by convolving these projected positions with a synthetic PSF. A synthetic 'detected' image, generated using the detected pixel coordinates of stars, accordingly centred and padded, is then cross-correlated with the synthetic reference image to provide the offset that is used to cross-match the stars. The pixel coordinates of a star $i$ in the detector plane $\left(\mathrm{x}_{i}, \mathrm{y}_{i}\right)$ are related to its sky position $\left(\mathrm{RA}_{i}\right.$, Declination $\left._{i}\right)$ via the expressions derived in K. Mierle \& D. W. Hogg (2007). ${ }^{5}$ The best-fitting solution is found using the bootstrap regression. The solution together with the individual matches are stored in the data base. Finally, the resulting FITS

\footnotetext{
${ }^{4}$ https://github.com/keflavich/image_registration

${ }^{5} \mathrm{http}$ ///astrometry.net/svn/trunk/documents/papers/wcs-tutorial/wcs.tex
}

World Coordinate System (WCS) is recorded to the registered FITS cubes.

A new data processing pipeline has been developed for photometry. After the standard bias subtraction and flat-fielding, photometric light curves of the objects of interest are derived based on differential photometry using field stars. Photometry is performed using both SEXTRACTOR (Bertin \& Arnouts 1996) and PYTHONPLOT (Jones, Scolnic \& Rodney 2015). In addition, the ability to phase fold light curves, important for periodic system, and produce animations from the data cubes is available.

To enable transient follow-up, image differencing software is available in the standard pipepline. The pipeline starts by constructing reference images from mosaiced frames from all-sky surveys; there are plug-ins available for SDSS, PS1, and ZTF. We astrometrically resample these template images using SWARP (Bertin et al. 2002) to cover the entire requested field. SWARP matches the template image to the science image from each epoch on a pixel by pixel level with an alignment typically accurate to $\approx 0.1$ pixels. We use HOTPANTS (Becker 2015) to photometrically match the template image to the science image. HOTPANTS divides each image into smaller 'stamps' and within each stamp, estimates the optimal kernel to convolve the template image into the science image. HOTPANTS then outputs a difference image which ideally, outside of transients, should be consistent with zero.

The code is publicly released and available from Github at the links given at the end of the paper.

\section{SYSTEM PERFORMANCE}

\subsection{Image quality}

To assess the image quality, we took images of Messier 13 (see left-hand panel of Fig. 4), also known as NGC 6205 or the Hercules globular cluster $(\mathrm{RA}=16: 41: 41.24, \mathrm{Dec} .=+36: 27: 35.5)$. On the right of Fig. 4 is a pair of histograms, both the point spread function shapes (A/B), and the full width at half-maximum (which assumes a Gaussian core) of the point spread function. The point spread function shape is represented by an ellipse with the lengths of the semimajor and semiminor axes denoted as A and B, respectively. The histograms indicate that the shape of the point spread functions are approximately circular, with the shapes A/B peaking near to 1 . This measurement is important as charge transfer inefficiency in the EM register can potentially cause image elongation (as can optical abberations, Rhodes et al. 2010; Israel et al. 2015). While we do not yet have measurements to differentiate between charge transfer inefficiency caused by the EM register and optical abberations, new techniques in investigating bulk trap limiting charge transfer efficiency can be used to thoroughly investigate signal smearing on the CCD (Bilgi 2019). These methods include the ability to characterize the emission time-scales of all relevant charge traps and measure their effects on data using the Extended Pixel Edge Response technique. Deferred charge measurements can be made accurate to $0.1 \mathrm{e}$ and furthermore, alternative clocking schemes are available to mitigate charge transfer trapping problems. This analysis can in principle be accomplished even while installed on the telescope, as long as one can expose the CCD to flat-field illumination and take dark frames.

In addition, the peak of the full width at half-maximum of the point spread function peaks between 6-8 pixels. At our plate scale, this indicates a seeing of approximately 1.5-2 arcsec, consistent with that expected at the 84-inch (Jensen-Clem et al. 2018). To determine the amount of distortion in the field, we use the PS1 

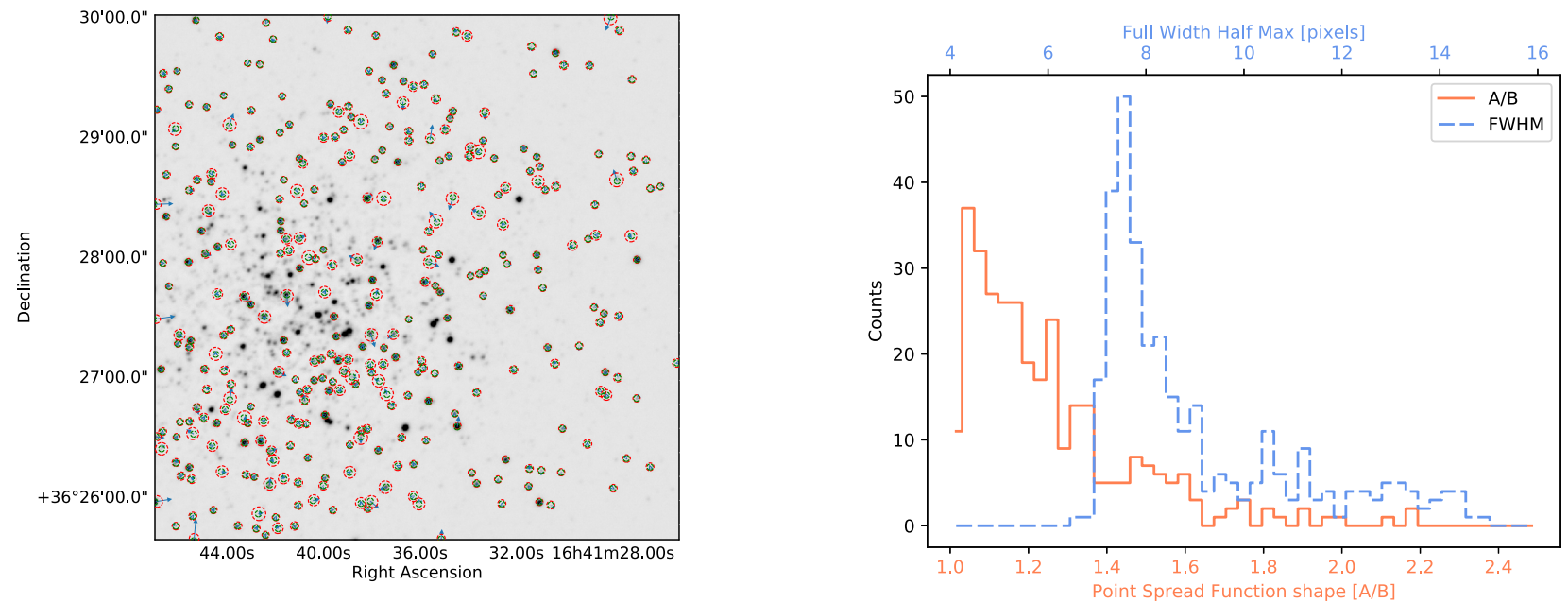

Figure 4. On the left is a KPED image of M13 in $r$ band. The green and red circles correspond to PS1 and KPED matched sources identified in the image. Blue arrows, $10 \times$ the size of the true separation, are also shown. On the right is a pair of histograms, both the point spread function shapes (A/B), and the full width at half-maximum of the point spread function.

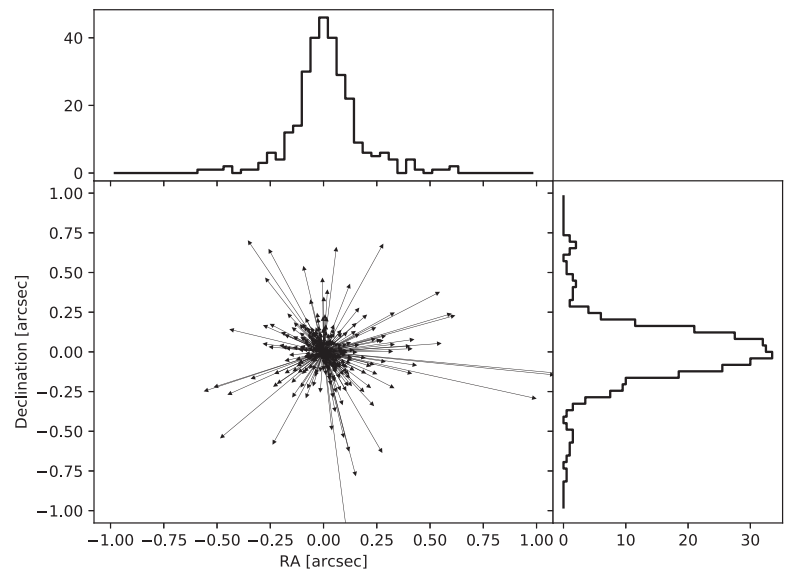

Figure 5. Quiver plot showing the distortion measured in the M13 image.

catalogue to match with KPED sources. In Fig. 5, a quiver plot shows the distortion measured in the M13 image. Separations are typically less than 0.5 arcsec, indicating excellent astrometry across the field.

\subsection{Pointing}

We generated a pointing model using 120 observations of bright Gaia DR1 stars $\left(6 \leq \mathrm{G}_{\mathrm{mag}} \leq 7\right)$. This was performed by first griding the sky in azimuth and elevation such that 120 points spread uniformly on the sky above an elevation of $20^{\circ}$ were chosen. To minimize the time required to image the sources, a greedy path was constructed to minimize the slew distances. During these observations, the telescope was first slewed to the coordinates of a star. At that point, if the star was identified in the field, the operator offset to the location of the star, and the successful find and its offset was noted. If not, the lack of success was noted, and the process was repeated for the next star. After the pointing model was produced from this data; the 'blind' pointing errors are within 1 arcmin, more than sufficient given the FOV of the instrument.

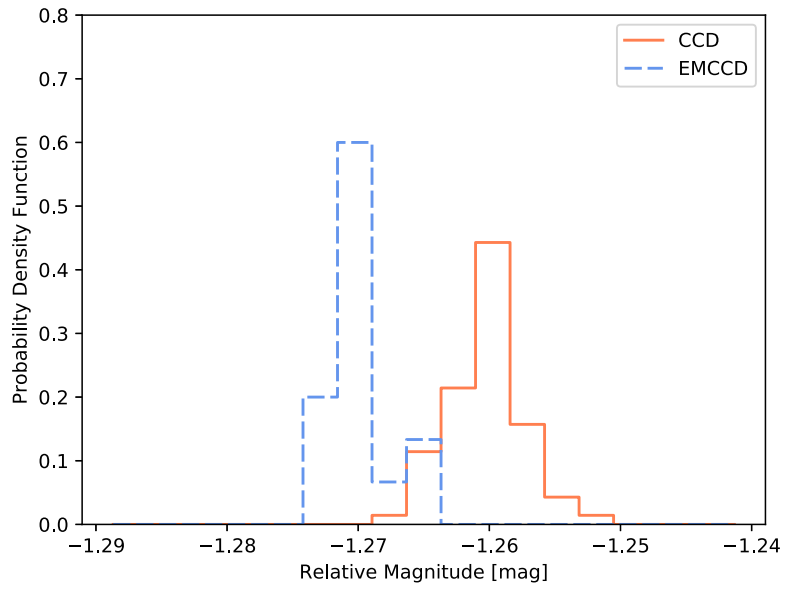

Figure 6. Photometry for $\mathrm{Hz} 44$ Photometry of HZ44 using both the conventional and EMCCD modes. The modes are consistent at the $0.01 \mathrm{mag}$ level. The data were taken in sets one after the other, and this difference likely arises from changes in airmass, seeing, and weather during this time.

\subsection{Photometric performance}

To assess both the photometric stability and compare between the conventional CCD and EMCCD modes in the camera, we took a series of images of $\mathrm{Hz} 44$, an European Southern Observatory (ESO) standard star $(\mathrm{RA}=13: 23: 35.37$, Dec. $=+36: 08: 00.0)$. It has a $V$-band magnitude of about 11.7 , which is bright enough for the statistical errors on the photometry to be small ( mag $_{\text {error }}<$ 0.01). By this comparison, the photometry of HZ44 using both the conventional and EMCCD modes is consistent to within $0.01 \mathrm{mag}$ (Fig. 6).

\subsection{Tracking}

To assess the performance of the tracking system, we use the same data set from Section 3.3 and explore the position of the star as a function of time over the hour of data. Fig. 7 shows the $X$ and $Y$ pixel positions as a function of image number over the hour of data. The drift is evident, and predominantly in right ascension (aligned with 


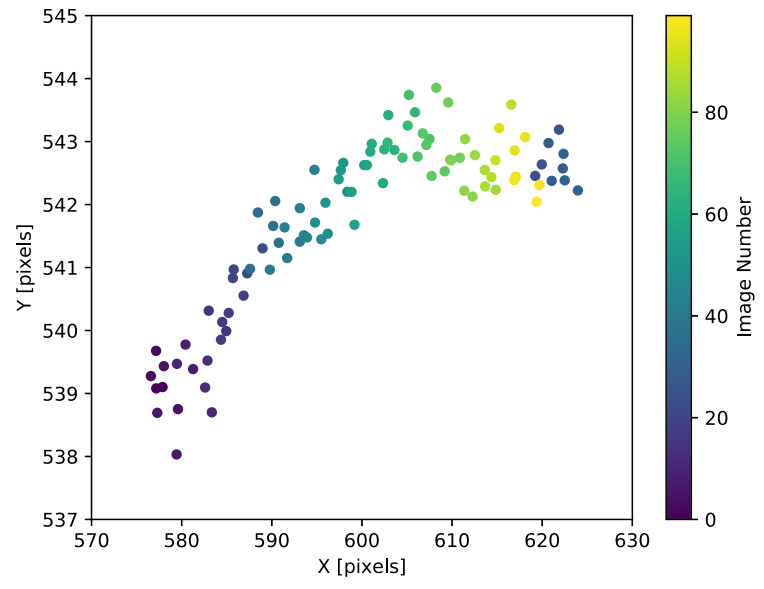

Figure 7. $\mathrm{X}$ and $\mathrm{Y}$ pixel positions as a function of image number for $\mathrm{Hz} 44$ in the EMCCD mode. The drift in $\mathrm{X}$ (corresponding to right ascension) is significantly larger than the drift in Y (Declination).

Table 3. KPED sensitivity and zero-points for the bands used for transient observations. The $5 \sigma$ limiting magnitudes are quoted for 300 -s observations (except for $I$ band, which is $5 \mathrm{~s}$ due to image saturations), which is the standard exposure time used. These zeropoints are computed for the conventional amplifier.

\begin{tabular}{lccc}
\hline Band & Limiting mag. & $\sigma_{\text {Limiting mag. }}$ & Zero-point mag. \\
\hline$r$ & 23.3 & 0.31 & 20.8 \\
$g$ & 23.7 & 0.48 & 21.3 \\
$U$ & 17.5 & 0.99 & 17.0 \\
$I$ & 16.2 & 0.49 & 16.1 \\
\hline
\end{tabular}

the $X$-axis). Over an hour, the star drifts about 40 pixels, which is approximately 9.6 arcsec per hour. While it makes observing single objects over long periods of time difficult, the effect on photometry is negligible due to the $10 \mathrm{~Hz}$ readout. This will be resolved in the future by adjusting the RA drive frequency.

\subsection{Astrometric solution}

The mean astrometric solution for KPED obtained using the best 36 successfully solved fields is given in Table 2. This solution is used as the default in the header files of KPED data before further reductions. Reduced data uses this solution as the basis for further refinement based on identified objects in the field.

\subsection{Zero-point and sensitivity}

For transient observations where absolute magnitudes are important, the zero-point of the field is calibrated using PAN-STARRS1 and Sloan Digital Sky Survey (SDSS) objects as standards. Given the coordinates of the target, an on-the-fly query to PAN-STARRS1 and SDSS retrieves the stars within the field that have a minimum of four detections in each band. To obtain the zero-point of images taken with the $g$ or $r$ filters, a direct comparison is made between the stars in the field and the standards in the retrieved catalogues. However, for the Johnson $U$ and $I$ bands, which are different from the PAN-STARRS1/SDSS $u$ and $i$ bands, the transformations of Jordi, Grebel \& Ammon (2006) are applied. Table 3 summarizes sensitivities for the commonly used bands for KPED with $300 \mathrm{~s}$ exposures, which is the standard adopted for transient observations.

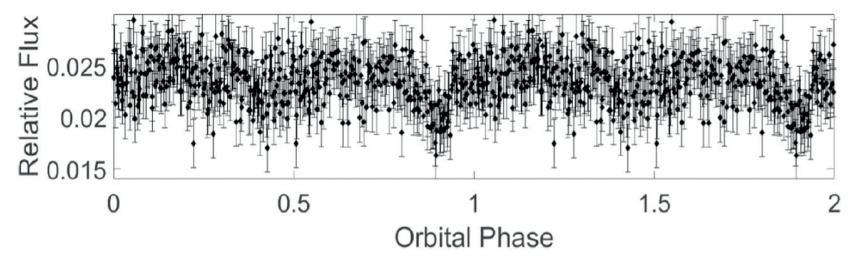

Figure 8. $g$-band light curve of J0651, folded to a period of $12.75 \mathrm{~min}$. Each data point corresponds to a 1-s integration. The data are reflected so as to showcase two identical cycles.

\section{SCIENCE RESULTS}

In this section, we present examples of three science cases described above: the characterization of eclipsing systems, pulsators, and photometry of ZTF transients. In the following light-curve analyses, we use the EMCCD mode with a gain of 300 , corresponding to an effective read noise of $<1 \mathrm{e}^{-}$rms. Because the objects highlighted here are bright, this configuration is suboptimal relative to the conventional mode with frame transfer, due to the multiplication noise penalty. Future analyses will highlight objects with deep eclipses, where the EMCCD is required to explore the very low SNR regime.

\subsection{Eclipsing systems}

To highlight the rapid cadence of light curves possible with KPED, we begin with observations of SDSS J065133.338+284423.37, known as J0651, which is a detached WD binary with a $12.75 \mathrm{~min}$ period (Brown et al. 2011; Hermes et al. 2012). It is the shortest period detached compact binary currently known. J0651 is special also for having both primary and secondary eclipses, ellipsoidal variations, and Doppler boosting, providing photometric markers whereby all orbital and system parameters can be measured. As a short-period system, it is useful for tracking the orbital decay due to gravitational wave radiation (Hermes et al. 2012), and one of the strongest known sources for LISA (Kupfer et al. 2018). Fig. 8 shows a 1-s cadence light curve of J0651, folded to a period of $12.75 \mathrm{~min}$, highlighting the primary and secondary eclipse for the system.

To show the potential use of high-cadence light curves for modelling, we observed the primary eclipse of PTF1J162528.61-003545.8, an eclipsing white dwarf-red dwarf system $(g=16.0)$ identified by PTF with an orbital period of $7.8 \mathrm{~h}$. Fig. 9 shows a $1 \mathrm{~h}$ light curve which covers the primary eclipse. Data were taken at $8 \mathrm{~Hz}$ and stacked to 1 -s images. We modelled the light curve using ELLC (Maxted 2016) and EMCEE (Foreman-Mackey et al. 2013). The bottom panel of Fig. 9 shows the difference between the data and model, with residuals around 5 per cent. This analysis showed that we can time the eclipse with a statistical uncertainty of $0.20 \mathrm{~s}$ and the sum of the scaled radii $\left(\left[R_{1}\right.\right.$ $\left.+R_{2}\right] / a$ ) and the ratio of the radii with a precision of $\approx 1$ per cent. The typical uncertainty on the individual measurements was 2.5 per cent.

\subsection{Ultrashort period pulsating stars}

One key advantage of a frame-transfer CCD lies in its ability to effectively eliminate readout times-enabling short exposures without sacrificing a substantial fraction of time to readout. Thus, an ideal science case for such an instrument is photometrically following up objects which require a rapid cadence of exposures 


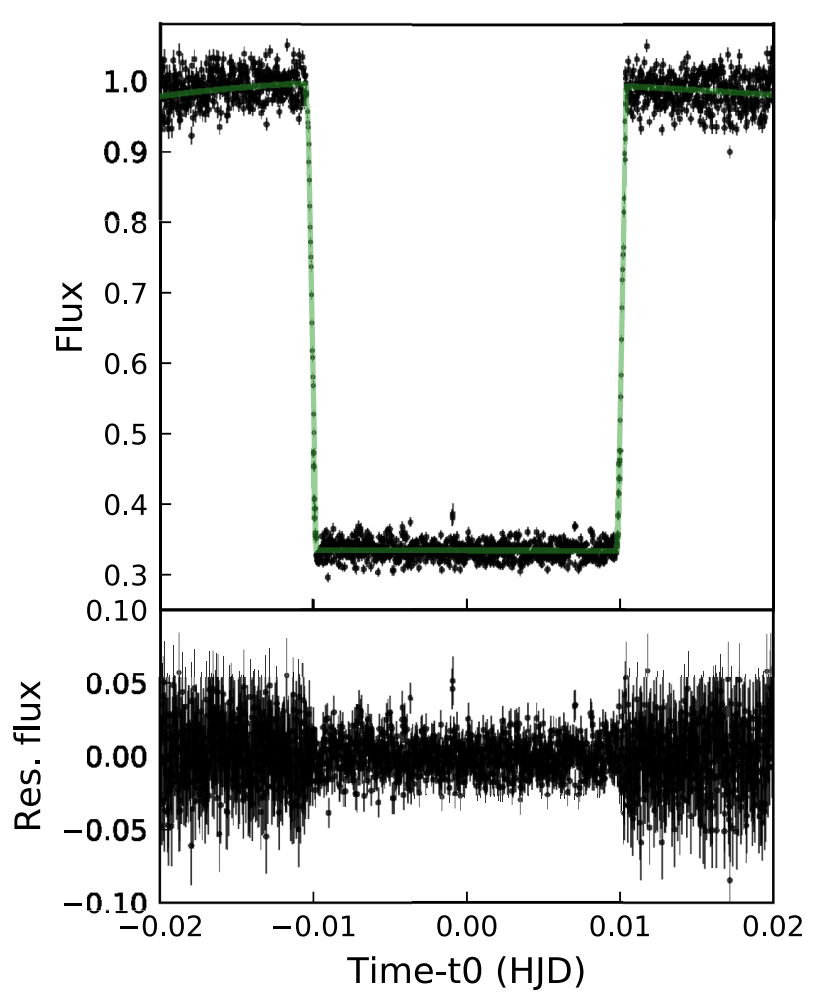

Figure 9. The top panel shows the $r$-band light curve of PTF1J162528.61-003545.8, showing the primary eclipse with the bestfitting model overplotted. The bottom panel shows the difference between the data and model, with residuals around 5 percent. Each data point corresponds to a 1-s integration.

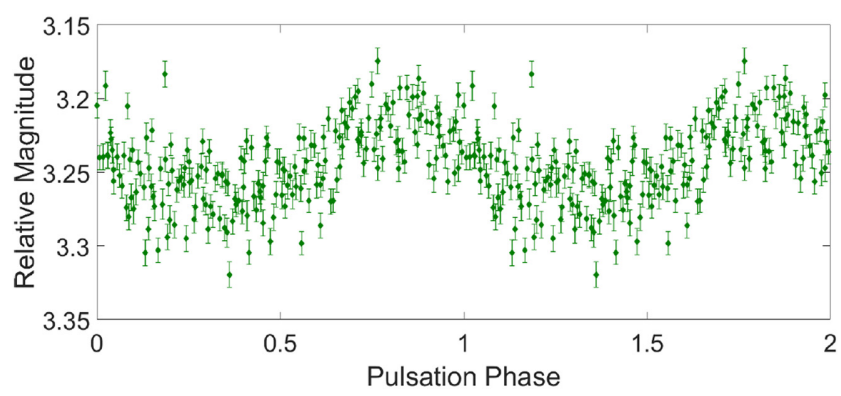

Figure 10. $g$-band light curve of PTF1J214022.55+262124.4 folded to a period of $4.8 \mathrm{~min}$. Each data point corresponds to a 1 -s integration. The data are reflected so as to showcase two identical cycles.

because they exhibit variable behaviour on very short time-scales. One such class of objects are pulsating WD and hot subdwarf stars, which typically exhibit periods on the order of $5 \mathrm{~min}$. To demonstrate KPED's capability in recovering the period waveform of one such object, we observed a newly discovered DB White Dwarf pulsator PTF1J214022.55+262124.4. Fig. 10 illustrates the phase folded light curve of the object, which clearly demonstrates the detection of the fundamental pulsation mode, which exhibits a period of only $4.8 \mathrm{~min}$. Pulsators serve as particularly interesting objects of study because pulsations can allow us to probe the interior structure of objects such as white dwarfs via astroseismology.

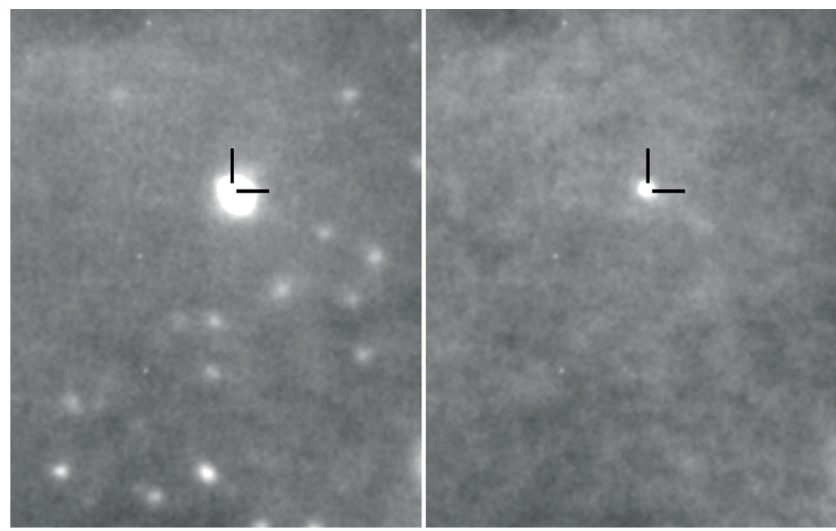

Figure 11. On the left is an image of ZTF18aalrxas in $r$ band. On the right is a difference image of the same (using an SDSS reference) resulting in a detection around $r=20.6 \mathrm{mag}$. The science frame has been degraded by the PSF of the SDSS reference (Fremling et al. 2016). There are visible multiplicative gradients in the background due to the lack of flat-fielding at the time the image was taken, which was the first night the instrument was on the telescope.

\subsection{ZTF transients}

With its robotic system and array of filters, KPED has the potential to contribute to the follow-up of objects found by all-sky surveys such as ZTF. For objects not necessarily in the high-cadence fields, or at depths not easily achieved by ZTF's nominal exposure times, dedicated follow-up is useful. As an example, we highlight ZTF18aalrxas in Fig. 11, which is an SN IIb with a double-peaked light curve recently identified by ZTF (and will be described fully in a separate forthcoming publication). Using difference imaging with ZTF images, a detection is made, reaching a depth around $r=22 \mathrm{mag}$. This object highlights the ability to effectively perform deep difference imaging using survey images as templates, which will be useful for transient follow-up.

\section{CONCLUSION}

As we have discussed, there is significant scientific value for a camera with rapid readout like KPED on a robotic telescope. With the dynamical time-scales of binary compact objects on the order of seconds, the ability to do high-cadence photometry with systems such as KPED is essential. In addition, the measurements of eclipses, transits, and occultations, which also occur on these time-scales, play an important role in constraining the physical parameters of the systems under study.

There are a number of potential routes to improve KPED. While the filter wheel allows for cycling between filters in order to get multiwavelength information, these measurements are both observationally inefficient and are unable to resolve colour variations on the time-scales shorter than constrained by the changing of filters. One possible solution is to use multiple channels, where multiple passbands are imaged simultaneously, which other systems have used to great effect (Dhillon et al. 2007; Dhillon et al. 2016; Harding et al. 2016)

In time-domain astronomy, spectroscopy is essential to classify transients discovered by wide FOV instruments. Due to the large sky localization areas of gravitational waves, neutrino events, and some gamma-ray bursts, the number of optical candidates can vary from dozen to hundreds. As these sources are usually rapidly fading, reducing follow-up times for objects is one of the key 
requirements to face the challenge of time domain astronomy. A robotic telescope equipped with a spectrograph and EMCCD allows for rapid response.

Next, a spectrograph would be useful for constraining parameters of eclipsing WD binaries (Hełminiak et al. 2017). For systems with eclipses of order $30 \mathrm{~s}$, spectra of that duration (or less) are required, otherwise other portions of the signal will be averaged. On the other hand, the EMCCD with the frame-transfer capability is capable of $1 \mathrm{~Hz}$ (and faster) readouts, and therefore spectra can be phase folded so as to get resolution on this scale. In addition, because spectra naturally disperse the light over a wider range of pixels, resulting in lower signal to noise in any given pixel, the low light levels are ideal for the use of EMCCDs.

KPED reduction code is available at: https://github.com/dmitryd uev/archiver-kped and https://github.com/mcoughlin/kp84.

\section{ACKNOWLEDGEMENTS}

MC is supported by the David and Ellen Lee Postdoctoral Fellowship at the California Institute of Technology. The KPED team thanks the National Science Foundation and the National Optical Astronomical Observatory for making the Kitt Peak 2.1m telescope available. We thank the observatory staff at Kitt Peak for their efforts to assist Robo-AO KP operations. The KPED team thanks the National Science Foundation, the National Optical Astronomical Observatory and the Murty family for support in the building and operation of KPED. In addition, they thank the CHIMERA project for use of the EMCCD. This work has made use of data from the European Space Agency (ESA) mission Gaia (https://www.cosmos.esa.int/gaia), processed by the Gaia Data Processing and Analysis Consortium (DPAC, https://www.cosm os.esa.int/web/gaia/dpac/consortium). Funding for the DPAC has been provided by national institutions, in particular the institutions participating in the Gaia Multilateral Agreement. The authors thank Dr Simon Mark Tulloch for reviewing the transcript and providing useful feedback. They also thank Pavan Bilgi and Roger Smith for a discussion of charge transfer inefficiency measurement techniques.

\section{REFERENCES}

Aasi J. et al., 2015, Class. Quantum Gravity, 32, 074001

Abbott B. P. et al., 2017, Phys. Rev. Lett., 119, 161101

Acernese F. et al., 2015, Class. Quantum Gravity, 32, 024001

Becker A., 2015, Astrophysics Source Code Library, record ascl:1504.004

Bellm et al., 2019, PASP, 131, 018002

Bertin E., Arnouts S., 1996, A\&AS, 117, 393

Bertin E., Mellier Y., Radovich M., Missonnier G., Didelon P., Morin B., 2002, in Bohlender D. A., Durand D., Handley T. H., eds, ASP Conf. Ser. Vol. 281, Astronomical Data Analysis Software and Systems XI. Astron. Soc. Pac., San Francisco, p. 228
Bilgi P., 2019, PhD thesis, California Institute of Technology

Breivik K., Kremer K., Bueno M., Larson S. L., Coughlin S., Kalogera V., 2018, ApJ, 854, L1

Brown W. R., Kilic M., Hermes J. J., Allende Prieto C., Kenyon S. J., Winget D. E., 2011, ApJ, 737, L23

Dekany S. et al., 2018, PASP submitted

Dhillon V. S. et al., 2007, MNRAS, 378, 825

Dhillon V. S. et al., 2016, in Evans C. J., Simard L., Takami H., eds, Proc. SPIE Conf. Ser. Vol. 9908, Ground-based and Airborne Instrumentation for Astronomy VI. SPIE, Bellingham, p. 99080Y

Dobbie P. D., Napiwotzki R., Lodieu N., Burleigh M. R., Barstow M. A., Jameson R. F., 2006, MNRAS, 373, L45

Foreman-Mackey D., Hogg D. W., Lang D., Goodman J., 2013, PASP, 125 , 306

Fremling C. et al., 2016, A\&A, 593, A68

Fuller J., Lai D., 2011, MNRAS, 412, 1331

Gaia Collaboration, 2016, A\&A, 595, A1

Gaia Collaboration, 2018, A\&A, 616, A1

GrahamM. J. et al., 2018, PASP , preprint (arXiv:1902.01945)

Harding L. K. et al., 2016, MNRAS, 457, 3036

Hełminiak K. G. et al., 2017, MNRAS, 468, 1726

Hermes J. J. et al., 2012, ApJ, 757, L21

Hermes J. J. et al., 2014, ApJ, 792, 39

Iben I. Jr, Tutukov A. V., 1984, ApJS, 54, 335

Israel H. et al., 2015, MNRAS, 453, 561

Ivezic Z., Tyson J. A., Allsman R., Andrew J., Angel R., 2008, in press

Jensen-Clem R., Duev D. A., Riddle R., Salama M., Baranec C., Law N. M., Kulkarni S. R., Ramprakash A. N., 2018, AJ, 155, 32

Jones D. O., Scolnic D. M., Rodney S. A., 2015, Astrophysics Source Code Library, record ascl:1501.010

Jordi K., Grebel E. K., Ammon K., 2006, A\&A, 460, 339

Kaplan D. L., 2010, ApJ, 717, L108

Kilpatrick C. D. et al., 2017, Science, 358, 1583

Kochanek C. S. et al., 2017, PASP, 129, 104502

Kupfer T. et al., 2018, MNRAS, 480, 302

Maxted P. F. L., 2016, A\&A, 591, A111

Morgan J. S., Kaiser N., Moreau V., Anderson D., Burgett W., 2012, in Stepp L. M., Gilmozzi R., Hall H. J., eds, Proc. SPIE Conf. Ser. Vol. 8444, Ground-based and Airborne Telescopes IV. SPIE, Bellingham, p. $84440 \mathrm{H}$

Rhodes J., Leauthaud A., Stoughton C., Massey R., Dawson K., Kolbe W., Roe N., 2010, PASP, 122, 439

Riddle R. L. et al., 2012, in Ellerbroek B. L., Marchetti E., Véran J. -P., eds, Proc. SPIE Conf. Ser. Vol. 8447, Adaptive Optics Systems III. SPIE, Bellingham, p. $84472 \mathrm{O}$

Saio H., Jeffery C. S., 2002, MNRAS, 333, 121

Savchenko V. et al., 2017, ApJ, 848, L15

Shappee B. J. et al., 2014, ApJ, 788, 48

Tonry J. L. et al., 2018, PASP, 130, 064505

Tulloch S. M., Dhillon V. S., 2011, MNRAS, 411, 211

Webbink R. F., 1984, ApJ, 277, 355

This paper has been typeset from a $\mathrm{T}_{\mathrm{E}} \mathrm{X} / \mathrm{LTT}_{\mathrm{E}} \mathrm{X}$ file prepared by the author. 